\title{
Sistema semiautomatizado para monitoramento topográfico
}

\author{
Semi-Automated System for Topographic Monitoring
}

Fabiani das Dores Abati Miranda ${ }^{1}$

Luis Augusto Koenig Veiga ${ }^{2}$

\author{
${ }^{1}$ Universidade Tecnológica Federal do Paraná - UTFPR. Estrada para Boa Esperança, km1,s/n, Câmpus Dois Vizinhos. \\ Cep 85660-000. Dois Vizinhos, PR, Brasil. \\ ${ }^{2}$ Departamento de Geomática. Universidade Federal do Paraná - UFPR. Centro Politécnico. Cep 81530-900. Curitiba, \\ PR, Brasil. \\ fabiani@utfpr.edu.br,kngveiga@ufpr.edu.br
}

Recebido em 06/06/2015 - Aceito em 14/03/2016

Received on 06/06/2015 - Accepted on 03/14/2016

\begin{abstract}
RESUMO - Deslizamentos de terras, colapsos de estruturas e até mesmo verificações de rotina, fazem com que o monitoramento de deslocamentos esteja constantemente presente em numerosos trabalhos de engenharia e pesquisas. $\mathrm{Na}$ área de pesquisa, os trabalhos são voltados à investigação de novas metodologias que associadas ao uso de equipamentos mais robustos e avançados, a uniformização e integração de dados, além da automação de diferentes etapas envolvidas, possibilitam soluções rápidas e eficientes, associando clareza e precisão à detecção e interpretação de resultados em um processo de monitoramento. Assim, neste contexto, desenvolveu-se um sistema semiautomatizado com apoio de estações totais, cujo objetivo é dar suporte ao monitoramento topográfico no que concerne à análise de objetos que sofrem deslocamentos, sendo que os cálculos e análise são realizados em tempo real. Para tanto, foram implementadas rotinas para a coleta, processamento, análise e representação gráfica dos dados. Optou-se pelo Método de Recessão no que se refere aos pontos de controle e pelo Método de Irradiação para a coleta dos pontos objeto. Experimentos de validação do sistema foram realizados e o mesmo apresentou-se viável para a utilização no monitoramento topográfico, possibilitando a repetibilidade e a série temporal dos pontos.
\end{abstract}

Palavras-chave: Automação, estação total, deslocamentos.

ABSTRACT - The collapse of structures, landslides and even routine check monitoring becomes a constant object of study in many engineering works and researches. The researches aims the investigation of new methods, latest equipment, data integration and automation of different steps involved in the work, always with the aim to detect and interpret results quickly and accurately. Within this context, it were developed a semi-automated system based on the use of total station, which will be used as support for topographic monitoring. The goal is to be able to perform an analysis of the object that undergoes displacement in real time, allowing greater flexibility and efficiency in the monitoring process. Thus, it were implemented routines for collecting, processing, analysis and graphic representation of the monitoring data. It were chosen the method of resection, with respect to the control points and the irradiation method for the measurement of object points. Validation experiments were performed and the systems proved to be feasible for use in topographic monitoring, providing the repeatability for time series of data.

Keywords: automation, total station, displacement.

\section{INTRODUÇÃO}

Embora os equipamentos disponíveis no mercado calculem as coordenadas de pontos em um monitoramento e possuam diversas funções para otimizar os trabalhos em campo, ainda se faz necessária a realização de diversos procedimentos para chegar a resultados que indiquem a variação da posição de um ponto ao longo do tempo.

Tais procedimentos englobam a escolha do instrumental, o modo como são distribuídos os pontos de apoio, os programas utilizados para coleta, processamento, verificação, ajustamento e análise dos dados coletados, a maneira como é realizada a comunicação e transferência dos dados entre o equipamento de medida e o computador, bem como o respectivo modo de armazenamento e visualização dos mesmos. O conjunto destes procedimentos caracteriza um sistema de monitoramento.

Parte do interesse no desenvolvimento desses sistemas automatizados origina-se na necessidade da determinação de deslocamentos em tempo real, principalmente quando está envolvido um grande volume de dados. Portanto, um Sistema automatizado ou semiautomatizado de monitoramento deve permitir o acompanhamento e estudo contínuo e sistemático do comportamento de fenômenos, eventos e situações específicas, cujas condições devem ser identificadas, avaliadas e comparadas em tempo real. 
Neste sentido, este trabalho apresenta um sistema semiautomatizado desenvolvido para o monitoramento (SAM) de pontos por técnicas topográficas em tempo real. Este sistema permite uma maior agilidade aos trabalhos de campo, minimizando erros no processo e possibilita ainda a conferência em tempo real dos dados observados, tornando-se uma ferramenta de suporte à decisão no que se refere ao monitoramento topográfico.

\section{PROJETO DO SISTEMA SAM - SISTEMA SEMIAUTOMATIZADO MONITORAMENTO TOPOGRÁFICO \\ PARA}

O SAM deve ser constituído levando-se em consideração os aspectos necessários para o monitoramento topográfico de objetos. Ao se analisar de forma sistêmica todos os detalhes que envolvem um projeto de controle de deslocamentos verificam-se que para obter medidas com alto grau de confiabilidade não é suficiente contar apenas com instrumentos de alta precisão e uma rede de pontos fixos de referência (MIRANDA, 2012). Neste planejamento deve-se definir ainda, uma metodologia de medição apropriada ao tipo de levantamento em questão (PACILÉO NETTO et al., 2002).

Deste modo, as metodologias empregadas foram definidas em função da necessidade de se ter a estação total em um sistema de referência consistente, sendo este definido por um conjunto de pontos. Isso foi possível empregando-se o Método de Recessão que segundo Kahmen e Faig (1988) o Método de Recessão consiste em determinar as coordenadas de um novo ponto ocupado pelo equipamento, a partir de observações de direções a estações de controle, bem como do conhecimento das coordenadas dessas estações. Em contrapartida a determinação das coordenadas dos pontos objeto, foi realizada por meio do Método de Irradiação. Método este utilizado por vários pesquisadores no que tange ao monitoramento de pontos (KAHMEN E FAIG, 1988; NEVES, 2008).

Assim sendo, todos esses elementos foram implementados em um programa com o intuito de gerenciar o SAM. Esse aplicativo foi desenvolvido na Linguagem Borland Delphi 7.0, em módulos cada qual responsável por um dos processos definidos a seguir:

a) Aquisição de dados - Recessão;

b) Banco de Dados;

c) Recessão;

1) Processamento e Análise dos dados;

2) Irradiação:

2.1) Aquisição de dados;

2.2) Processamento e análise;

2.3) Representação gráfica;

2.4) Relatório;

3) Representação Gráfica;

4) Relatório.

Cabe salientar que o programa não gerencia a coleta de dados, as medidas devem ser executadas pelo operador, o programa recebe, armazena e processa os dados, sendo os resultados apresentados na forma de relatórios e gráficos. Devido a isto, o sistema foi denominado de semiautomatizado.

\subsection{Módulo de aquisição de dados}

Este módulo é responsável pela aquisição de dados. É por meio deste que todas as observações podem ser coletadas e armazenadas para posterior processamento e análise.

Mas, para que o programa possa receber e armazenar as observações é necessário realizar a configuração dos parâmetros de comunicação da estação total, bem como do computador.

Com os dois equipamentos configurados, a porta de comunicação pode ser aberta, os pontos podem ser observados e, por conseguinte, o recebimento das observações poderá ser realizado. Cabe salientar que o programa foi configurado para o recebimento de códigos no formato especificado pela fabricante do equipamento. Neste caso, utilizou-se a estação total Leica TC 403L e o formato empregado é o Geo Serial Interface (GSI), mais especificamente o GSI 8.

O programa recebe o código enviado pelo equipamento e armazena as informações no banco de dados. Neste módulo são coletadas apenas observações referentes ao Método de Recessão. O processo de aquisição de pontos irradiados (pontos objeto) é realizado dentro do módulo irradiação seguindo os mesmos passos.

\subsection{Módulo de banco de dados}

No SAM as observações coletadas em campo são armazenadas no banco de dados, isto é, cada observação é automaticamente armazenada em uma das tabelas do banco de dados no momento de sua aquisição, informações tais como: direções horizontais, ângulos zenitais, distâncias inclinadas, série de medição, época, hora, data e demais informações sobre o levantamento em questão.

Sendo assim, verificou-se a necessidade de um módulo que permitisse a conferência, edição ou verificação dessas observações coletadas, para tanto, foi criado o módulo banco de dados.

Nesse, o acesso a essas observações é feito por meio da busca por nome do ponto de interesse e/ou época. Definidas estas informações o mesmo faz uma varredura em seus dados e retorna valores, tais como: direções, ângulos, distâncias. Ainda nesse módulo é possível realizar a edição e/ou remoção de pontos e inclusão de informações sobre o levantamento.

\subsection{Módulo de recessão}

O módulo Recessão é responsável pelo processamento e análise dos dados, geração de gráficos e relatórios, elementos que auxiliarão nas avaliações e tomadas de decisão.

\subsubsection{Processamento e análise}


As observações são avaliadas de forma automática e análise de deslocamento é obtida em tempo real. Para tanto, os seguintes passos devem ser executados no que diz respeito ao processamento e análise das mesmas:

a) Definir ponto de Ré, precisões horizontal e vertical e tolerância do par conjugado;

b) Verificar par conjugado: este passo inclui o cálculo das séries coletadas de direções horizontais, dos ângulos zenitais e distâncias inclinadas. Esse módulo realiza a verificação dessas séries, se as mesmas estão dentro da precisão estipulada;

c) Média e desvio-padrão: são calculadas a média e desvio-padrão das séries.

d) Partida: nesta etapa são definidas as informações referentes a partida, se os pontos de controle possuem coordenadas conhecidas ou se o levantamento é realizado no sistema local. Para realizar o ajustamento quatro pontos de controle deverão ser medidos.

e) Calcular Recessão: nesta etapa aplica-se o método de Recessão para determinar as coordenadas da estação ocupada.

f) Verificar a estabilidade das estações de controle: deve ser possível verificar se existe ou não variação nas distâncias entre as estações de controle, sendo uma forma de verificar se as mesmas sofreram ou não deslocamentos.

g) Ajustar: nesta etapa as coordenadas da estação ocupada são ajustadas e as matrizes variânciacovariância calculadas para cada época, possibilitando assim, analisar a qualidade do ajustamento.

h) Testes estatísticos: Neste passo, as observações são testadas para verificar se as mesmas possuem ou não erros grosseiros. Caso possuam, uma intervenção manual será necessária.

\subsubsection{Relatórios}

O módulo Relatório foi desenvolvido para que o SAM pudesse ter suas observações, bem como todos os resultados dos processamentos e análises apresentados na tela do computador e também de forma impressa. Ele permite que o processamento e análise dos dados de uma ou mais épocas sejam salvos em arquivos de texto (.txt) ou visualizados na tela do programa.

\subsubsection{Representação gráfica}

Uma das necessidades no monitoramento é a garantia de que o ponto onde a estação está instalada não sofre variações durante o período do monitoramento.

Assim sendo, após as coordenadas finais serem calculadas em cada época de observações, gráficos podem ser gerados para auxiliar na análise e interpretação dos deslocamentos resultantes.

Neste módulo são gerados quatro gráficos que permitem avaliar como se comportam as coordenadas $\mathrm{X}$, $\mathrm{Y}, \mathrm{X}$ ajustada e $\mathrm{Y}$ ajustada, todas em função das épocas.
Também é possível avaliar a discrepância entre as coordenadas observadas e as correspondentes ajustadas em dois gráficos adicionais.

\subsubsection{Irradiação}

Dentro do módulo de Recessão existe uma etapa denominada Irradiação. Essa se faz necessária para que sejam determinadas as coordenadas dos pontos objeto, ou seja, dos pontos distribuídos na área de interesse, para os quais deseja-se verificar se existe ou não deslocamento.

Determinadas as coordenadas dos pontos objeto, o programa permite ainda quantificar a diferença, existente ou não, entre épocas distintas. A época inicial (denominada de época 1) é sempre tida como época base, isto é, admite-se que os dados obtidos nesta sejam os dados de referência sobre os quais serão feitas as comparações. O mesmo ocorre no método de Recessão. Com o intuito de verificar a qualidade dos resultados, o programa calcula a propagação de erros, bem como os elementos da elipse de erros.

Após os cálculos, todos os resultados são apresentados na tela do programa. Estes podem ainda ser salvos em arquivos de texto (.txt). O programa permite a geração de dois gráficos. Eles referem-se à diferença de coordenadas dos pontos objeto em relação à época 1 , ou seja, serão plotadas informações de variações de coordenadas por épocas de observação.

\section{SAM}

O programa SAM foi desenvolvido tendo como principais objetivos: coletar as observações de forma semiautomatizada; armazenar os dados em um banco de dados relacional; executar o processamento dos dados de forma automática, gerar gráficos de apoio à análise dos resultados; ajustar utilizando o MMQ e no caso dos pontos objetos realizar a propagação de erros, bem como calcular os elementos das elipses de erros.

Como pode ser observado na Fig. 1, o programa apresenta em sua tela principal três módulos de operação. Cabe salientar que o programa foi implementado de acordo com o planejamento apresentado na metodologia.

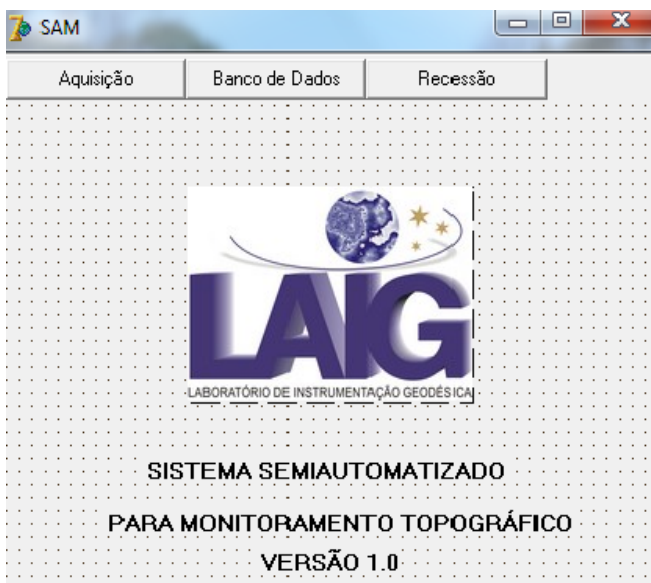

Figura 1 - Tela principal do SAM.

Fonte: MIRANDA (2012). 
O módulo Aquisição (Fig. 2) é o primeiro a ser selecionado. Este é responsável pela coleta de observações para aplicação do método de Ressecção. Nele são realizadas todas as configurações de comunicação, para que o programa possa se comunicar com a estação total, por meio da porta serial. Após a configuração ser realizada, o passo seguinte consiste na abertura da porta serial, para que a coleta de dados seja iniciada. A porta serial só será fechada no término das observações.

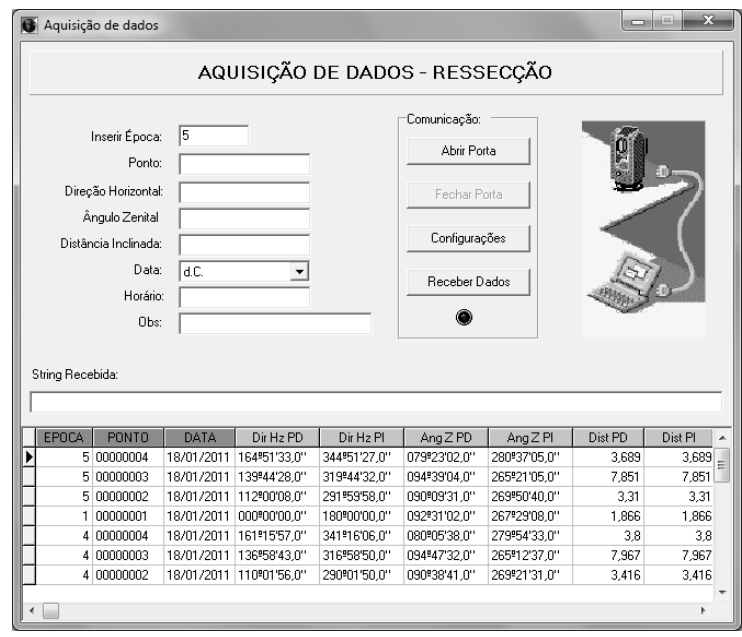

Figura 2 - Programa SAM - Aquisição de dados. Fonte: MIRANDA (2012).

Cabe ressaltar que a época deve ser informada no início do monitoramento, para que o programa possa armazenar as observações de forma correta no banco de dados e que a coleta de parâmetros ambientais: pressão, temperatura seca e úmida, deve ser realizada de forma manual.

Já o módulo banco de dados (Fig. 3) deve ser utilizado quando deseja-se realizar alguma edição e/ou remoção das observações coletadas. Ele apresenta em sua tela todas as informações relacionadas a um ponto préselecionado, isto é, pode apresentar em sua tela qualquer ponto coletado, com suas séries de observações, para cada época do monitoramento.

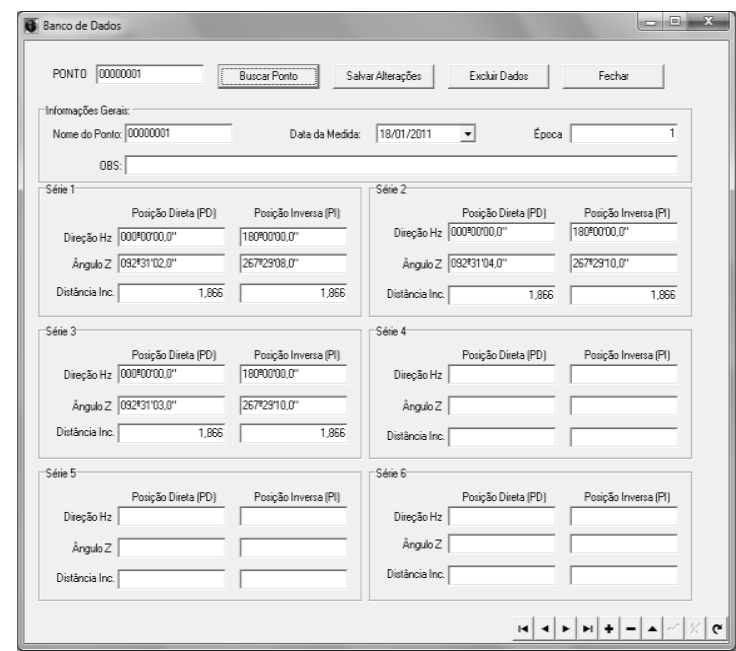

Figura 3 - Programa SAM - Banco de Dados. Fonte: MIRANDA (2012).
De posse das observações, parte-se para o procedimento de verificação das observações, o qual é realizado dentro do módulo Recessão. Ele permite que o cálculo dos pares conjugados de cada série, verificação das tolerâncias pré-definidas, cálculo das médias das séries, bem como do desvio-padrão, seja realizado de forma automática.

Após a verificação deve-se definir a partida: se serão introduzidas coordenadas de partida e se será realizado o ajustamento; se somente serão introduzidas as coordenadas das estações de controle sem a necessidade de realização do ajustamento; ou ainda se será utilizado um sistema local com ou sem ajustamento. $\mathrm{O}$ ajustamento só poderá ser selecionado, caso quatro estações de controle sejam observadas. É o número mínimo a ser considerado para a realização do mesmo, pois para calcular as coordenadas ajustadas da estação ocupada pelo MMQ empregando-se a Recessão é necessário observar mais de três direções, para tanto, no mínimo quatros pontos de controle devem ser observados.

Caso o sistema local seja selecionado, as coordenadas das estações de controle serão calculadas.

Por fim, o programa utiliza as fórmulas do método recessão para o cálculo das coordenadas da estação ocupada. Após isso, elas são ajustadas pelo MMQ, utilizando o Modelo Paramétrico e é realizada uma análise da qualidade do ajustamento, aplicando os testes estatísticos, Data Snooping, Tau, Q-Quadrado, são aplicados para a análise e detecção de erros. Outros elementos calculados pelo programa são as distâncias entre as estações de controle. Estas podem ser analisadas em épocas distintas como forma de verificar se essas estações sofrem deslocamentos.

Todos os resultados são apresentados na tela do programa (Fig. 4), os gráficos podem ser gerados, bem como os relatórios.

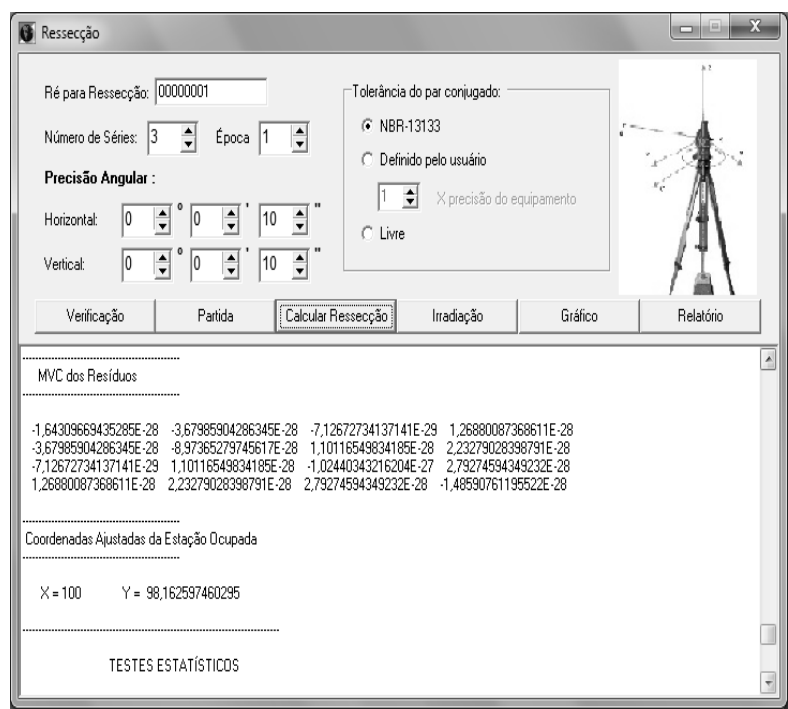

Figura 4 - Programa SAM - Recessão.

Fonte: MIRANDA (2012).

Determinadas as coordenadas da estação ocupada, parte-se para a obtenção das coordenadas do ponto objeto (ponto a ser monitorado), por meio da aplicação do 
método de Irradiação. Para tanto, deve ser realizada uma nova coleta de observações, seguindo-se o mesmo procedimento descrito anteriormente na coleta de dados da Recessão. Toda vez que acontece uma nova coleta, o programa realiza novamente a verificação das mesmas, analisando se estão dentro das tolerâncias pré-definidas, depois calcula suas médias e desvio-padrão de suas séries (Fig. 5).

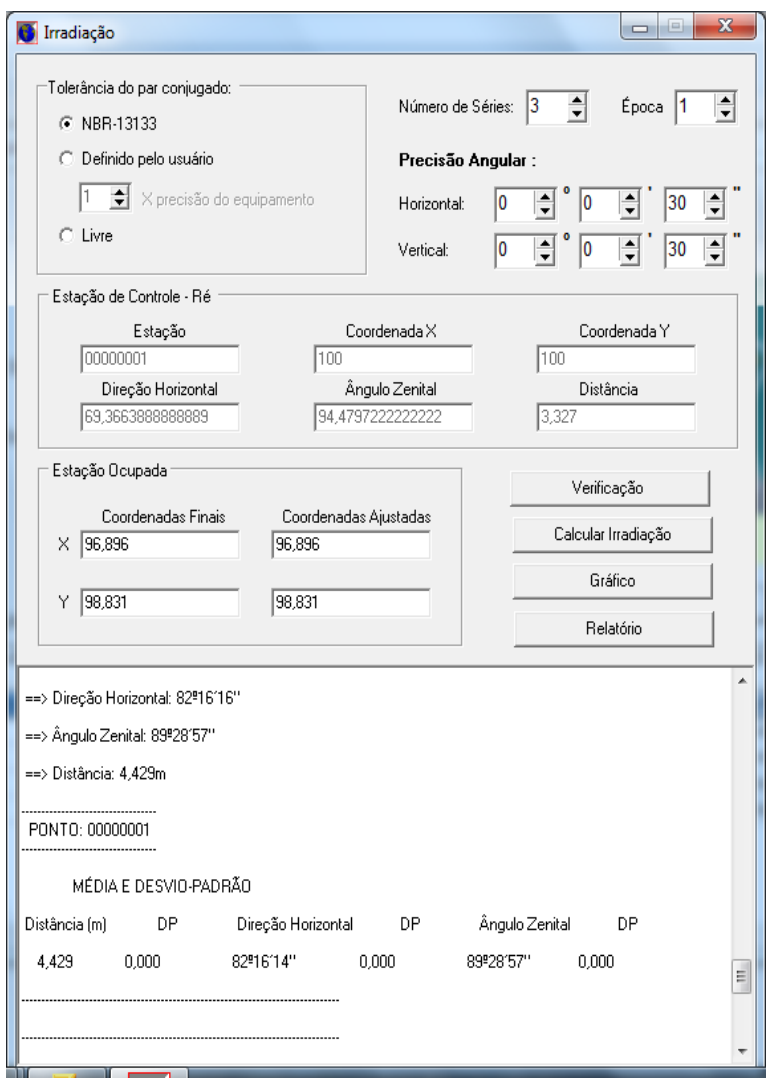

Figura 5 - Programa SAM - Irradiação.

Fonte: MIRANDA (2012).

Por fim as coordenadas do ponto objeto são calculadas em função das coordenadas e observações da estação de ré utilizada na Recessão e das coordenadas da estação ocupada. Quando são realizadas mais épocas de observações o programa informa as discrepâncias entre as coordenadas obtidas entre essas épocas distintas, bem como é gerado um gráfico dessas diferenças.

\section{TESTE DE VALIDAÇÃO DO SAM}

O experimento foi realizado no Laboratório de Instrumentação Geodésica (LAIG - UFPR), tendo como objetivo simular o deslocamento de um objeto e quantificá-lo. Neste experimento utilizou-se a estação total Leica TC 403L para realizar as medições de direções, ângulos e distâncias, com precisão angular de $10 "$ e linear de $3 \mathrm{~mm}+3 \mathrm{ppm}$, e um Interferômetro laser Hewlett Packard modelo 5508A, com precisão, nas condições em que se encontra instalado no Laboratório de Instrumentação Geodésica da Universidade Federal do Paraná LAIG-UFPR, de $\pm 0,010 \mathrm{~mm}$ (FAGGION et al.,
2008), para determinar com acurácia o afastamento entre os pontos objetos.

Foram distribuídos quatro pontos de controle no LAIG e sobre o Interferômetro posicionou-se um ponto objeto, materializado por um prisma refletor.

Como o objetivo era controlar o deslocamento e verificar se o sistema conseguiria quantificá-lo, optou-se por locar o ponto objeto em duas posições distintas no interferômetro, isto é, na primeira época de observação um prisma foi colocado em uma posição inicial, registrada no interferômetro com o valor $100.00 \mathrm{~mm}$ (distância em relação ao zero do sistema de medida, ao longo dos trilhos do interferômetro). Após, foram realizadas três séries de observações de direções, ângulos e distâncias, caracterizando assim primeira posição do ponto objeto.

Em seguida, deslocou-se o mesmo para a posição $105.00 \mathrm{~mm}$, sendo também realizadas as séries de observações. Definiu-se assim a segunda posição do ponto objeto. Desta forma, o deslocamento obtido à partir das observações com o SAM será comparado com o deslocamento executado com o interferômetro, igual a $5 \mathrm{~mm}$. Este levantamento com o auxilio do interferômetro permitiu controlar o deslocamento do ponto objeto. As medições que caracterizaram a segunda época desenvolveram-se seguindo o mesmo processo, sendo modificado somente o local onde a estação total foi instalada.

\subsection{Resultados}

$\mathrm{Na}$ Tab. 1, podem ser visualizadas as coordenadas das quatro estações de controle calculadas com as observações da Época 1. Estas foram tidas como coordenadas de partida para o cálculo na segunda época.

Tabela 1 - Coordenadas calculadas das estações de controle.

\begin{tabular}{ccc}
\hline \multirow{2}{*}{ ESTAÇÃO } & $\mathbf{X}$ & $\mathbf{Y}$ \\
\hline $\mathbf{1}$ & 100,000 & 100,000 \\
$\mathbf{4}$ & 95,910 & 95,300 \\
$\mathbf{3}$ & 96,023 & 93,732 \\
$\mathbf{2}$ & 100,366 & 94,121 \\
\hline
\end{tabular}

Na Tab. 2, são apresentados os ângulos horizontais obtidos em função da direção horizontal da estação de controle 1 .

Tabela 2 - Ângulos Horizontais

\begin{tabular}{|c|c|c|}
\hline & \multicolumn{2}{|c|}{ ÂNGULOS HORIZONTAIS } \\
\hline & Época 1 & Época 2 \\
\hline$\alpha$ & $126^{\circ} 13^{\prime \prime} 43^{\prime \prime}$ & $139^{\circ} 37^{\prime} 58^{\prime \prime}$ \\
\hline$\beta$ & $120^{\circ} 21^{\prime} 14^{\prime \prime}$ & $131^{\circ} 45^{\prime} 8^{\prime \prime}$ \\
\hline
\end{tabular}

Após o cálculo destes, parte-se para o cálculo das coordenadas da estação ocupada. As coordenadas ajustadas da estação ocupada para as duas épocas pode ser visualizada na Tab. 3 e a variação entre as mesmas pode ser observada na Fig. 6. 
Tabela 3 - Coordenadas da estação ocupada.

\begin{tabular}{ccc}
\hline EST. & \multicolumn{2}{c}{ COORDENADAS (m) } \\
OCUP & Época 1 & Época 2 \\
\hline $\mathbf{X}$ & 96,896 & 96,946 \\
$\mathbf{Y}$ & 98,831 & 98,227 \\
\hline
\end{tabular}

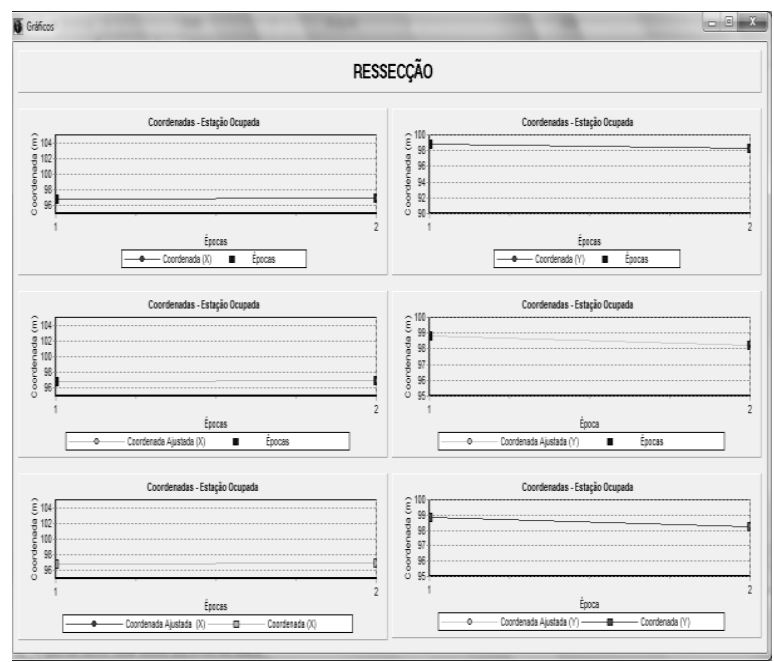

Figura 6 - Coordenadas da estação ocupada x Épocas de Monitoramento (Gerado pelo SAM)

Fonte: MIRANDA (2012).

Vale ressaltar que o programa aplicou automaticamente os testes Data Snooping e Tau, para um nível de significância de 5\%, com intuito de avaliar se as observações possuíam ou não erros grosseiros. Os resultados mostraram que as mesmas estavam isentas de erros. Um outro teste aplicado foi o Qui-quadrado, este por sua vez, comprovou a qualidade do ajustamento.

A partir das coordenadas da estação ocupada, calcularam-se pelo método de irradiação, as coordenadas dos pontos objetos para as duas épocas de observação (Tab. 4). O ponto objeto 1 , refere-se ao prisma locado na posição $100.00 \mathrm{~mm}$ registrada pelo interferômetro e o ponto 2, a posição $105.00 \mathrm{~mm}$ registrada pelo mesmo. Além disso, realizou-se a propagação de erros para as mesmas.

Tabela 4 - Coordenadas calculadas do ponto objeto.

\begin{tabular}{cccccc}
\hline \multirow{2}{*}{ PTOS OBJETO } & \multicolumn{4}{c}{ COORD. PONTO OBJETO (m) } \\
& & Época 1 & $\sigma$ & Época 2 & $\sigma$ \\
\hline \multirow{2}{*}{ P1 } & $\mathbf{X}$ & 101,285 & 0,003 & 101,287 & 0,003 \\
& $\mathbf{Y}$ & 99,427 & 0,001 & 99,428 & 0,001 \\
\multirow{2}{*}{ P2 } & $\mathbf{X}$ & 101,285 & 0,003 & 101,287 & 0,003 \\
& $\mathbf{Y}$ & 99,422 & 0,001 & 99,423 & 0,001 \\
\hline
\end{tabular}

Ao se comparar os resultados das duas épocas de monitoramento pode-se observar que as discrepâncias encontradas para o ponto P1 e P2 foram iguais ou inferiores a $2 \mathrm{~mm}$, portanto, dentro da precisão do equipamento. Já na questão do deslocamento controlado, verifica-se que o SAM conseguiu detectar e quantificar o mesmo, isso pode ser comprovado analisando-se as diferenças entre as coordenadas do P1 e P2 na época 1, onde em X não houve deslocamento e em Y detectou-se $5 \mathrm{~mm}$, exatamente o valor pré-estabelecido com interferômetro no monitoramento. Nos valores obtidos na época 2, verifica-se que o mesmo ocorreu, ou seja, foi detectado um deslocamento de $5 \mathrm{~mm}$ em $\mathrm{Y}$, entre os pontos P1 e P2.

Após definir as posições de pontos e as suas precisões, partiu-se para a representação gráfica tanto da posição relativa dos pontos quanto de suas precisões, sendo que esta representação pode ser visualizada pela elipse de erros (Fig. 7). Vale ressaltar que o SAM calcula todos os elementos da elipse de erros, mas não gera um gráfico das mesmas.

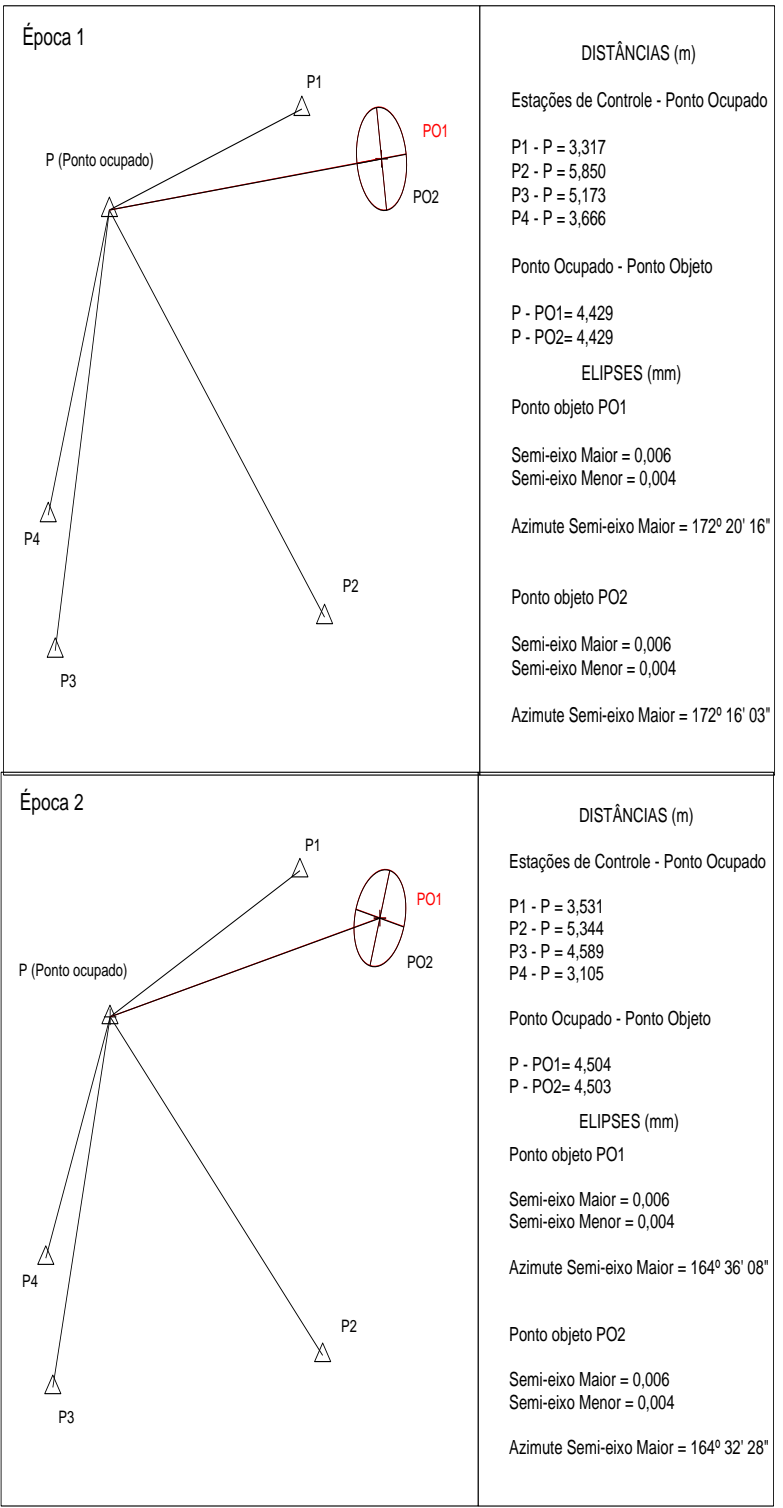

Figura 7 - Distribuição dos Pontos e Elipse de Erros

Fonte: MIRANDA (2012)

\section{CONCLUSÃO}

No SAM é possível trabalhar com séries de observações, processamento dos dados, aplicação de testes estatísticos, geração de gráficos e relatórios. O mesmo opera de forma semiautomatizada o que garante rapidez no processamento e análise dos resultados. 
Avaliando-se esses elementos constatou-se que o SAM desenvolvido atendeu a todos os requisitos básicos necessários, o que pode ser comprovado no teste de validação. Apresentou ainda, resultados satisfatórios quanto da sua utilização no monitoramento topográfico, pois respondeu com resultados coerentes a realidade em questão, bem como permitiu que os mesmos fossem apresentados logo após a realização das medições - a ideia principal do desenvolvimento do sistema.

Assim, a área de Geodésia aplicada ao monitoramento passa a ter disponível mais uma ferramenta para auxiliar no monitoramento de pontos. Ferramenta esta de fácil utilização, que ajudará na tomada de decisão em tempo real, durante o próprio decorrer do monitoramento.

\section{RECOMENDAÇÕES}

- Acoplar ao sistema sensores meteorológicos, o que possibilitará que as distâncias sejam corrigidas, em tempo-real, das influências dos fatores atmosféricos; Implementar outros testes estatísticos para avaliação dos deslocamentos obtidos;

- Habilitar o sistema para ser utilizado com diferentes estações totais;

- Implementar a determinação e análise da componente altimétrica Z;

- Implementar outros modelos de ajustamento ao SAM, bem como outros métodos de medição;

- Realizar experimentos considerando a circunferência perigosa, tendo em vista que a mesma possibilita um melhor posicionamento do ponto sobre o qual

- o equipamento está instalado; Modificar o SAM para que o mesmo possa controlar de forma remota a coleta de dados, e que está possa ser feita de forma totalmente automatizada.

\section{REFERÊNCIAS BIBLIOGRÁFICAS}

FAGGION, P. L.; SILVA, T. F.; ANDOLFATO, S. H. D.; SANTOS, D. P. dos. Calibração de mira horizontal de invar e sua aplicação na determinação de distâncias com alta precisão. II Simpósio Brasileiro de Ciências Geodésicas e Tecnologias da Geoinformação. Recife- PE, 2008.

KAHMEN, H.; FAIG, W. Surveying. Berlin: Walter de Gruyter, 1988.

MIRANDA, F. D. A. Monitoramento Topográfico em Tempo-Real com a Utilização de um Sistema SemiAutomatizado. Tese (Doutorado em Ciências Geodésicas) - Setor de Ciências da Terra, Universidade Federal do Paraná, Curitiba, 2012.

NEVES, R. V. $\begin{gathered}\text { Processamento e análise de } \\ \text { levantamentos } \\ \text { geodésicos aplicado }\end{gathered}$
$\begin{aligned} & \text { monitoramento de estruturas civis. Dissertação } \\ & \text { (Mestrado em Ciências Geodésicas) }\end{aligned}$
Ciências da Terra, Universidade Federal do Paraná, Curitiba, 2008.

PACILÉO NETTO, N.; BLITZKOW, D.; BUENO, R. F. ; FONSECA JR, E. S. da; MAZZILLI, C. E. N.; LACHAPELLE, G. Monitoramento de Deslocamentos em Estruturas com GPS. In: 3 ASAMBLEA HISPANO-PORTUGUESA GEODESIA E GEOFISICA, 2002, Valencia. Proceedings...Valencia : UPV, 2002. 